\title{
VARIABLE PITCH HELICAL DRIVE IN-PIPE ROBOT
}

\author{
Tao Ren, ${ }^{*}$ Qingyou Liu, ${ }^{* *}$ Yonghua Chen,${ }^{* * *}$ and Shouhong Ji****
}

\begin{abstract}
Pipelines are extensively used to transport water, oil, and gas for daily human activities and industrial production. An in-pipe robot is necessary to prevent fluid leakage accident by regularly detecting the quality status of a pipeline. Given the complex paths and shapes of pipes, an in-pipe robot cannot easily pass through curved pipes. In this study, a novel variable pitch helical drive in-pipe robot is introduced. The variable pitch adjusting mechanism makes switching the robot between three motion patterns (normal, variable speed, and turning) easy. The proposed robot can pass through curved pipes actively by controlling the helical angle of each driving wheel separately. The space spiral kinematic trajectories of the passive typical and the active novel helical in-pipe robots are compared. A prototype is developed to verify the validity of the proposed structure and function.
\end{abstract}

\section{Key Words}

In-pipe robot, variable pitch, helical drive, curved pipe, turning motion

\section{Introduction}

Pipe safety problems have become a critical issue as global pipeline construction rapidly develops. Over the past decades, the number of pipeline fluid leakage and explosion accidents has been increasing sharply because of the lack of safety awareness in effective measures for pipeline inspection. Pipeline accidents lead to serious casualties, economic losses, and even ecological damages.

Several methods (e.g., manual excavation, device detection on ground, and pipeline pig inspection) should be implemented to decrease pipeline accidents by inspecting pipelines regularly [1]-[3]. At present, in-pipe robot inspection is the most widely studied method for detecting

* School of Mechatronic Engineering, Southwest Petroleum University, Chengdu, China; e-mail: rtswpu@qq.com

** Key Laboratory of Fluid and Power Machinery, Ministry of Education, Xihua University, Chengdu, China; e-mail: liuqy666@yahoo.com

*** Department of Mechanical Engineering, The University of Hong Kong, Hong Kong, China; e-mail: yhchen@hku.hk

**** Zhejiang Zhenergy Natural Gas Operation Co., Ltd, Zhejiang, China; e-mail: jishh@gas.zhenergy.com.cn

Recommended by Prof. Chaomin Luo

(DOI: 10.2316/Journal.206.2016.3.206-4774) and repairing pipelines [4]-[6]. However, some problems and difficulties exist in real applications. Oil and gas pipeline grids come in various complex sizes and shapes. Using curved pipes, branch pipes, and fittings is inevitable. In-pipe robots, which can adapt well to changes in pipe shape and size, (particularly curved pipes) are urgently required.

In recent years, many famous scholars have conducted extensive research on the use of in-pipe robots for complex pipeline inspection, particularly curved pipes. These robots have several different structures and driving approaches.

(1) Active wheel steering: A stable and smooth fuzzy steering controller is constructed to operate inside pipes [7]. The steering wheel is installed in front of the robot. It steers actively inside curved pipes via fuzzy control. However, passing through complex pipes highly depends on fuzzy control rules, which are subjective.

(2) Self-deformation of a robot to adapt to pipe bending: A mobile robot "KANTARO" has been proposed for sewer pipe inspection [8]. The wheels of this robot can adapt to pipe bends passively through the passiveactive intelligent moving mechanism. Another miniature pipe inspection robot has been presented; this robot can also move through extremely small pipes (with up to $41 \mathrm{~mm}$ inner diameter, which is equal to the driving wheel diameter of the robot) [9]. A robot called "SPRING" has been developed as a new piping inspection robot that can travel through gas pipes with reducers and elbows [10]. Complex structures and controlling strategies are always necessary when applying this turning principle.

(3) Passing through curved pipes via a differential mechanism: A wheel-type pipeline robot with environmental self-adaptation capability and differential motion function has been developed [11]. This robot can automatically regulate the speed of driving wheels through the tri-axial differential mechanism to improve the locomotion compliance. MRINSPECT VI++ with differential gear mechanism is designed to be driven with single actuator at elbow type pipelines without additional control efforts [12].

(4) Modular in-pipe robot with hinge or flexible connection: A robot called "OmniTread OT-4" can climb 
over obstacles that are considerably higher than its size; it can also propel itself inside pipes with different diameters [13]. However, this robot is difficult to control when travelling through large-diameter pipes, elbows, or T-branches. The authors [14] have produced a modular inchworm helical in-pipe robot. A conic coil spring that allows extension and retraction is placed between helical drives. This in-pipe robot has considerably good mobility when turning a bend passively because of its flexible body (conic springs provide the inchworm robot with a compliant mechanism).

However, the curve passing function of in-pipe robots remains difficult to achieve. If a single module of the aforementioned modular in-pipe robot is slightly long, then getting stuck in curves is easy. Most turning principles, including those in the previous works of the authors, are passive [15], [16]. Thus, the structure of the robot itself cannot compliantly solve the problem of passing through curved pipes. Some of these principles are difficult to apply in auto-control in pipe bends and in adapting to such hostile environments.

In the present study, we present a new design of a variable pitch helical drive in-pipe robot that can pass through curved pipes actively by calculating and controlling the helical angle of each driving wheel separately. Given that driving wheels have different moving speeds, this robot can be concentric with the centre of the pipe when passing through curves. It can also prevent itself from being stuck in the pipe. The moving flexibility of this robot is also improved. The proposed robot provides new ideas for future research on robot trafficability characteristics in complex pipelines.

\section{Basic Design Concept}

Eight basic robot types that can be used in pipelines are available: pig, wheel, caterpillar, wall-press, walking, inchworm, helical, and snake types [17], [18]. With the recent demand for miniaturization and simplification, many studies have focused on helical-type in-pipe robots, which can be used in the petroleum, chemistry, and nuclear fields [19]-[21]. First, a helical-type robot has a simple structure with only one driving motor, and thus, it is easy to miniaturize. This type of robot also demonstrates high reliability in hostile pipeline environments. Second, a helical-type robot can pass through pipes with torques smaller than those of typical wheeled robots [16]. It can also conduct full pipe diameter coverage contact measurement without requiring any auxiliary rotation motor.

However, a helical-type in-pipe robot experiences some difficulties when passing curves. The driving wheels of typical helical-type in-pipe robots have the same inclination angle that provides the same rotation speed. This structure frequently causes eccentricity and stoppage in elbows. In this study, we investigate a novel variable pitch helical drive in-pipe robot that has driving wheels with different helical angles. This robot exhibits good trafficability and can remain at the centre even when passing through curved pipes.

Figure 1(a) shows a typical screw drive in-pipe robot. The three driving wheels are in the same helical angle,

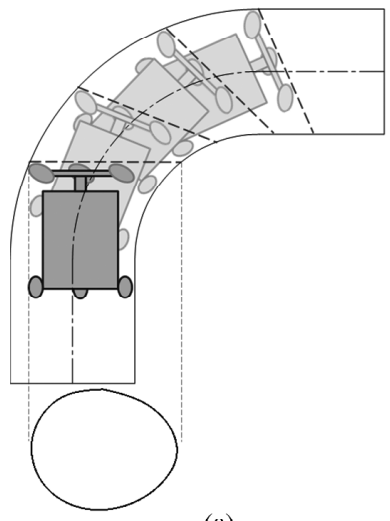

(a)

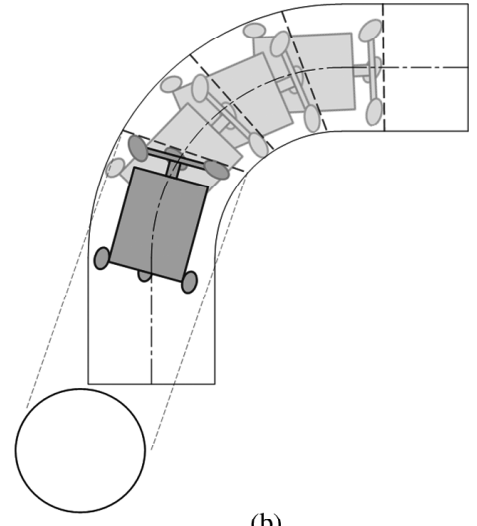

(b)
Figure 1. Two situations in pipe: (a) typical screw drive in-pipe robot and (b) variable pitch helical drive in-pipe robot.

and thus, they exhibit the same speed. Consequently, the moving direction of the typical screw drive in-pipe robot is forward and straight. When passing curved pipes, only the pipe-bending shape can passively change the orientation of the robot. Thus, the curved trajectory does not coincide with the pipe centre line. The plane of the robot wheels is egg shaped, which is irregular for wheels to pass through curved pipes. The contact pressure between the pipe inner wall and the wheel in the outer side of the pipe is excessively large, whereas the status of the wheel in the inner side of the pipe is always slippery. Consequently, the robot can easily get stuck in the pipeline.

Figure 1(b) shows a variable pitch helical drive in-pipe robot. The helical angles of the driving wheels can be adjusted when the robot is moving in pipelines. The three driving wheels can be adjusted in different helical angles to achieve various moving speeds. When passing curved pipes, the outer wheel accelerates, whereas the inner wheel decelerates. Accordingly, the central moving trajectory of the variable pitch helical drive in-pipe robot is curved, and it coincides with the pipe centre line. Therefore, the plane of the robot wheels is circular and nearly along the radial direction of the pipe. This mechanism, which is called "passing through curved pipes actively," is the goal that we intend to attain in this research.

\section{Variable Pitch Helical In-Pipe Robot Design}

\subsection{Structure}

The variable pitch helical in-pipe robot consists of a power unit, a driving unit, a centring unit, and a variable pitch unit. The power unit (a DC source or a built-in battery) provides power to the robot. The driving unit provides the robot with driving force. The centring unit makes the robot concentric with the pipelines. The variable pitch unit can adjust the inclination angle (helical angle) to change the driving force and moving speed of the robot. Springs are installed on the driving wheels and the centring wheels to adjust the diameter of the pipe. The driving wheels press on the inner pipe wall to produce the driving force. The concrete structure is shown in Fig. 2. 


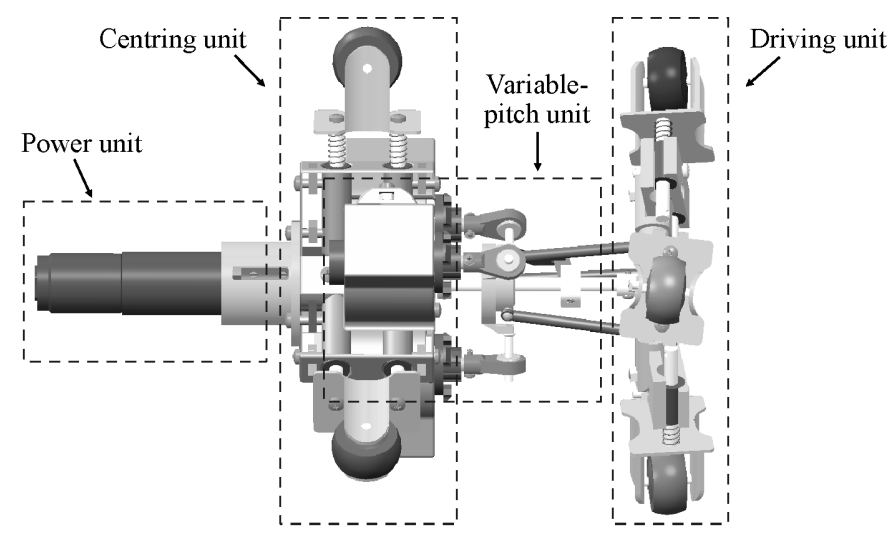

Figure 2. Variable pitch helical in-pipe robot structure.

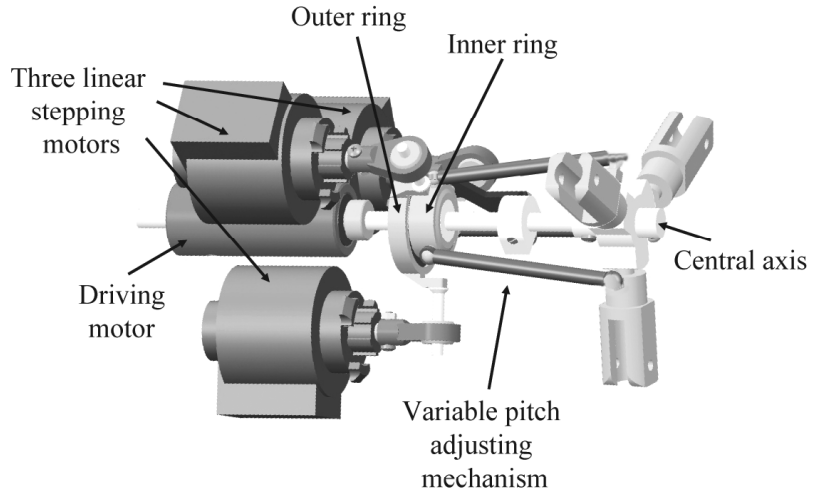

Figure 3. Structure overview of the variable pitch adjusting mechanism.

\subsection{Principle of Variable Pitch}

The pitch of the moving trajectory of the driving wheels can be adjusted by changing the helical angle of each driving wheel separately. Thus, the three driving wheels can achieve different moving speeds along the forward direction of the robot. The different speeds of the three driving wheels make the robot flexible, particularly when passing elbows.

The structure overview of the variable pitch adjusting mechanism is shown in Fig. 3. The driving motor rotates the driving wheel frame. Thus, the driving wheels rotate under the influence of the friction force between the driving wheels and the inner pipe wall. Figure 4 shows one of the pitch adjusting mechanisms. The driving wheel is initially parallel to the cross section of the pipe (Fig. 4(a)). The linear stepping motor is controlled to change the length of its output axis. Then, the inner and outer rings on the central axis move or tilt. The connecting rod pushes the driving wheel to tilt at a new angle. The inclination angle of the driving wheel can be changed accordingly (Fig. 4(b)). Thus, the pitch of the helical moving trajectory of a driving wheel can be changed. If the three helical angles of the driving wheels are controlled in the same value, then the robot is in variable speed motion. Otherwise, it is in turning motion. The details are described in Section 3.3.

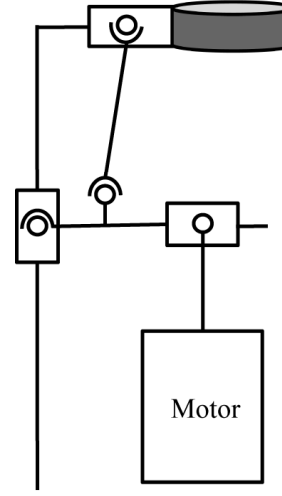

(a)

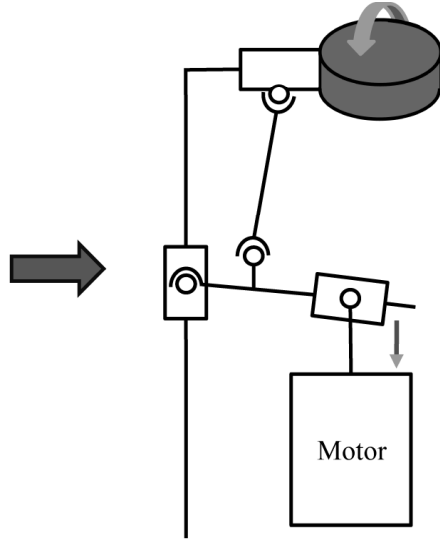

(b)
Figure 4. Schematic of working principle of the variable pitch adjusting mechanism: (a) the initial state and (b) variable pitch state.

\subsection{Motion}

After the driving motor starts, torque is transmitted to the central axis. Then, the motor driving shaft rotates along with the central axis. Given that the driving wheels press on the inner pipe wall, they rotate in a helical path and cause the whole in-pipe robot to move forward. The guiding wheels press on the inner pipe wall and play an important role in centring and balancing the counter torque. The variable pitch mechanism is applied to the driving unit to adjust the inclination angles of the driving wheels.

The variable pitch helical in-pipe robot has three motion patterns.

(1) Normal motion: The outer ring remains stationary with the centring unit. The inner ring rotates along with the driving unit. The robot maintains a constant speed and a helical angle.

(2) Variable speed motion: The output axes of the linear stepping motors are adjusted to be in the same length. Then, the inner and outer rings move together in parallel, which results in a new and the same inclination angles to the driving wheels. Thus, the speed of the robot can be changed without changing the rotation speed of the driving motor.

(3) Turning motion: The output axes of the linear stepping motors are adjusted with different lengths. Then, the outer and inner rings are inclined together, which results in different inclination angles to the driving wheels. Thus, the pitch of the helical moving trajectory of the driving wheels can be changed when passing curved pipes. The robot can pass through a curved pipe actively.

\section{Kinematics Analysis}

Whether a robot can achieve helical motion in a pipe and pass through curved pipes successfully highly depends on the working principle of the robot and on the inner shape of the pipe. The kinematics models of two different helical motions in curved pipes are built in this section. 


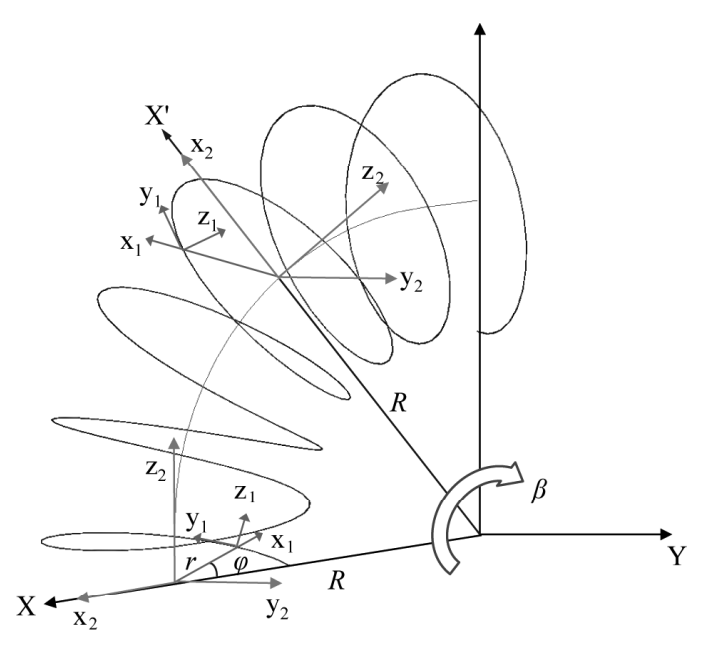

Figure 5. Kinematics model of constant pitch.

\subsection{Constant Pitch Space Spiral Trajectory in Curved Pipes}

Typical screw drive in-pipe robots nearly have the same structure that contains a stator and a rotator [22]-[24]. This type of robot can pass through straight pipes with high reliability. However, when passing curved pipes, the driving wheels of such robot have the same inclination angle. The space spiral trajectories of the driving wheels are in the same constant pitch [25]. Therefore, the robot passively passes through curved pipes. The kinematics model of one trajectory is shown in Fig. 5 .

Three coordinates are established: one is global coordinate $X Y Z$, whereas the other two are local coordinates $x_{1} y_{1} z_{1}$ and $x_{2} y_{2} z_{2}$. A curved pipe that rotates $90^{\circ}$ around the $Y$-axis is parallel to the $X Z$ plane. At first, coordinate $x_{1} y_{1} z_{1}$ rotates around the $z_{2}$-axis with rotation angle $\varphi$ and radius $r$. A constant inclination angle $\alpha$ (the inclination angle of a driving wheel) of the $y_{1}$-axis exists. Then, coordinate $x_{2} y_{2} z_{2}$ rotates around the $Y$-axis with rotation angle $\beta$ and radius $R$. Thus, the final trajectory is a helical line that exhibits bending and upward motion. The constant pitch space spiral trajectory equation is established as follows:

$$
\left\{\begin{array}{l}
x=(R+r \cdot \cos \varphi) \cdot \cos \beta \\
y=r \cdot \sin \varphi \\
z=(R+r \cdot \cos \varphi) \cdot \sin \beta
\end{array}\right.
$$

where $\beta$ and $\alpha$ exhibit the following relationship:

$$
\beta=\frac{r \varphi \tan \alpha}{R}
$$

\subsection{Variable Pitch Space Spiral Trajectory in Curved Pipes}

The variable pitch helical in-pipe robot introduced in this study can change the inclination angles of the three driving

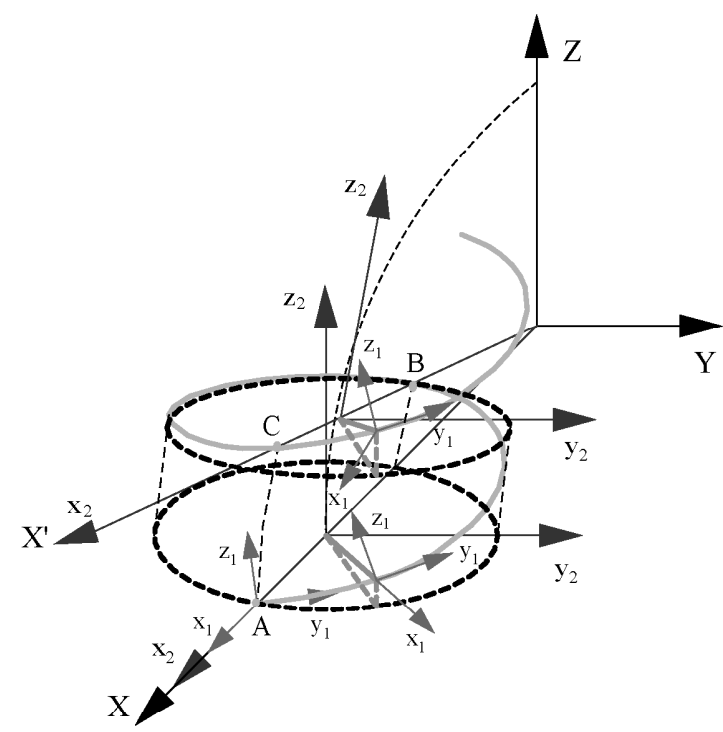

Figure 6. Kinematics model of variable pitch.

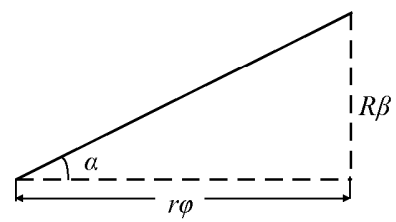

(a)

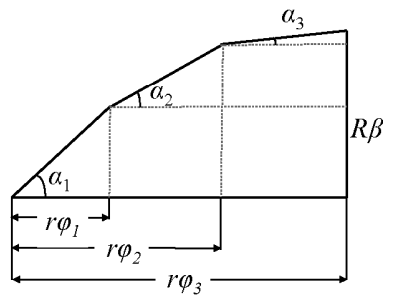

(b)
Figure 7. Diagrammatic sketch of two types: (a) constant pitch and (b) variable pitch.

wheels independently. In particular, when one of the driving wheels is passing the outer side of the curved pipe, its helical angle should have the maximum value among the three driving wheels. By contrast, if it is passing the inner side of the curved pipe, its helical angle should have the minimum value. Accordingly, the outer wheel moves fast, whereas the inner wheel moves slowly. The turning motion is active, and the robot achieves an excellent elbow-through performance. Figure 6 shows the kinematic model.

Three coordinates are established, and the parameters are the same as those presented in Section 4.1. The relative motions of the three coordinates are also similar to those given in Section 4.1. The difference is that helical angle $\alpha$ is variable. The helical angle of outer point $A$ on the helical trajectory has the maximum value. The forward movement of local coordinate $x_{1} y_{1} z_{1}$ results in the decrease of the helical angle to zero at point $B$ on the inner side. Then, the helical angle increases to the maximum value at point $C$ on the outer side. Point $C$ starts a second cycle.

The different helical angles of the constant pitch and the variable pitch are shown in Fig. 7. The rotation angle $\beta$ shown in Fig. 7(a) can be calculated using (2). The helical angle $\alpha$ shown in Fig. 7(b) is variable, and is assumed to be in the following periodic value:

$$
\alpha=\frac{k}{2} \cos \varphi_{i}+\frac{k}{2}
$$


where $\varphi$ is equal to $2 \pi n t_{i}$, and $i$ values range from 1 to the total number $m$ of the collected data. In this study, $k$ denotes the maximum helical angle, and $n$ denotes the rotation speed. The helical angle, which continuously changes over time, is divided into a limited number of constant values. Then, arc lengths, which correspond to each helical angle, are accumulated. Thus, we have the final arc length $L$ of the curved trajectory of the $z_{2}$-axis:

$$
L=\sum_{i=1}^{m} 2 \pi n r\left(t_{i}-t_{i-1}\right) \cdot \tan \alpha
$$

Rotation angle $\beta$ can be obtained as follows:

$$
\beta=\left|\frac{L}{R}\right|
$$

The final variable pitch space spiral trajectory equation can be derived by substituting (3), (4), and (5) into (1).
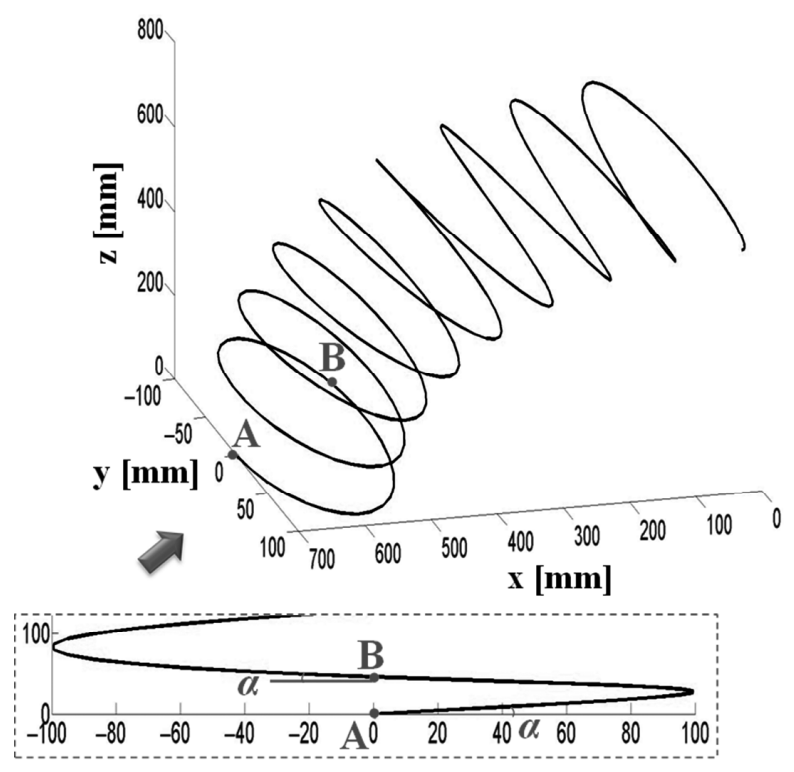

(a)

\subsection{Space Spiral Trajectory Comparison}

The parameters are defined as $R=600 \mathrm{~mm}, r=100 \mathrm{~mm}$, $n=0.1 \mathrm{r} / \mathrm{s}$, and $t=0$ to $220 / 3 \mathrm{~s}$. The maximum helical angle of the variable pitch is $20^{\circ}$, whereas its minimum helical angle is 0 . The helical angle of the constant pitch should be $10^{\circ}$ (the average value ranges from $20^{\circ}$ to $0^{\circ}$ ) to make the two trajectories comparable. The two trajectories are shown in Fig. 8. Outer point $A$ is the starting point of the helical trajectory, and inner point $B$ is on half the period of rotation angle $\varphi$. The helical angles $\alpha$ of points $A$ and $B$ are both $10^{\circ}$ in Fig. 8(a). However, Fig. 8(b) shows that the helical angle $\alpha$ of point $A$ is $20^{\circ}$, whereas that of point $B$ is 0 . The comparison of the constant pitch and the variable pitch is shown at the front view of Fig. 9. Figure 9(a) is in constant pitch, and the helical angle is constant. Figure 9(b) is in variable pitch, and the helical angle decreases from the maximum value to 0 and then increases from 0 to the maximum value in every period.
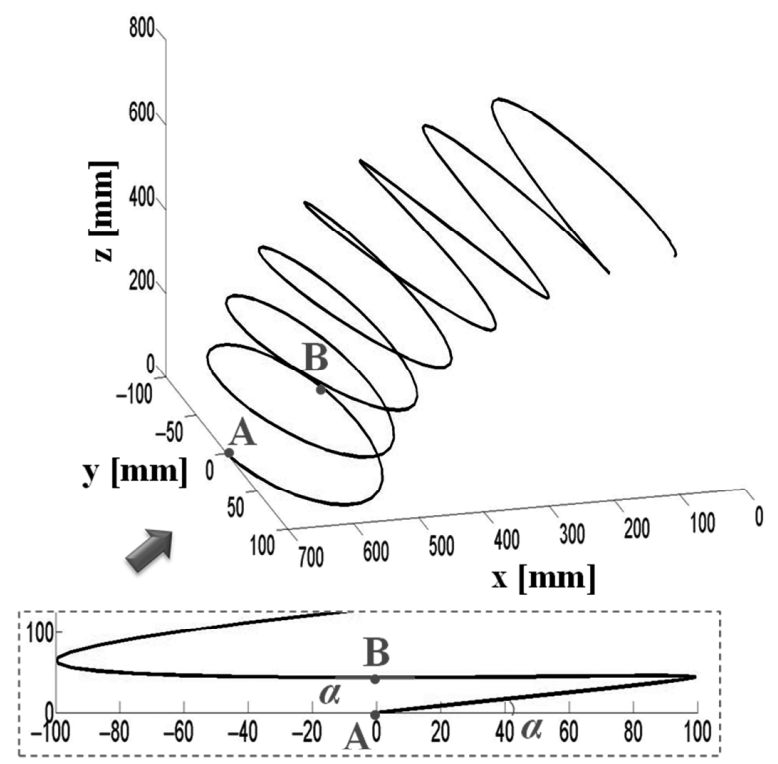

(b)

Figure 8. Two helical trajectories: (a) constant pitch and (b) variable pitch.

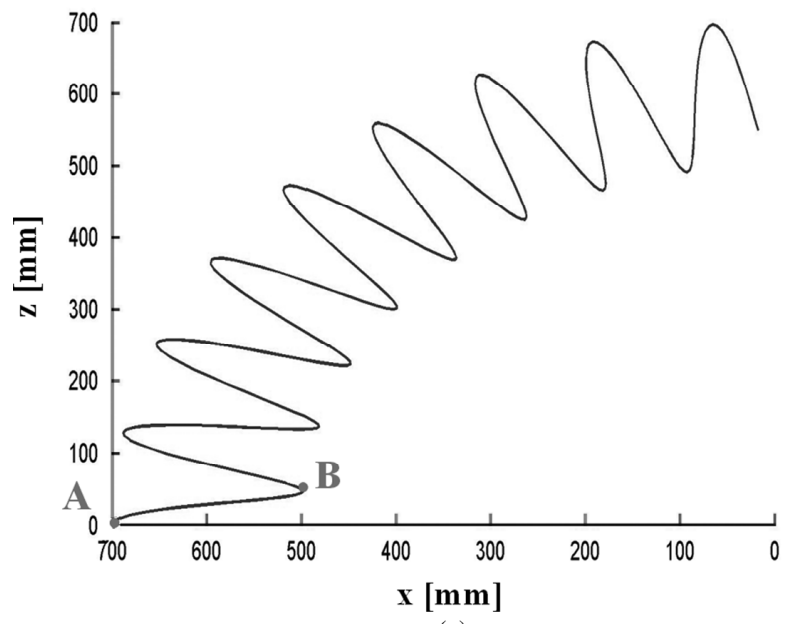

(a)

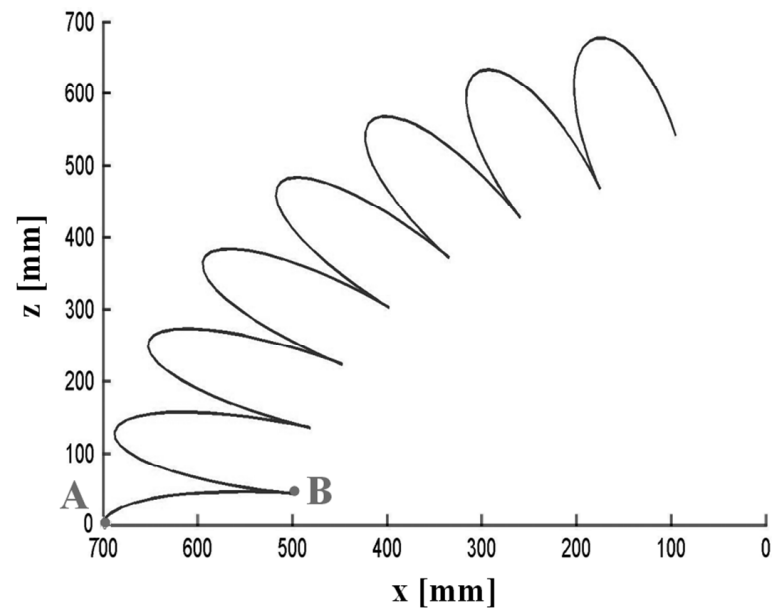

(b)

Figure 9. Front view of two helical trajectories: (a) constant pitch and (b) variable pitch. 


\section{Prototype and Experiments}

A prototype of the variable pitch helical in-pipe robot is developed, as shown in Fig. 10, to validate the variable pitch structure and motion characteristics. The prototype is $230 \mathrm{~mm}$ long. It can adapt to pipe diameters ranging from $160 \mathrm{~mm}$ to $210 \mathrm{~mm}$.

As illustrated in Fig. 11, the MCU communicates with a computer via the WiFi module. The MCU controls three linear stepping motors and a DC motor. The driving wheel frame is driven by the DC motor. A current sensor is used to measure driving current. Real-time posture of the prototype can be measured by the three-axis gyroscope of MPU 6050.

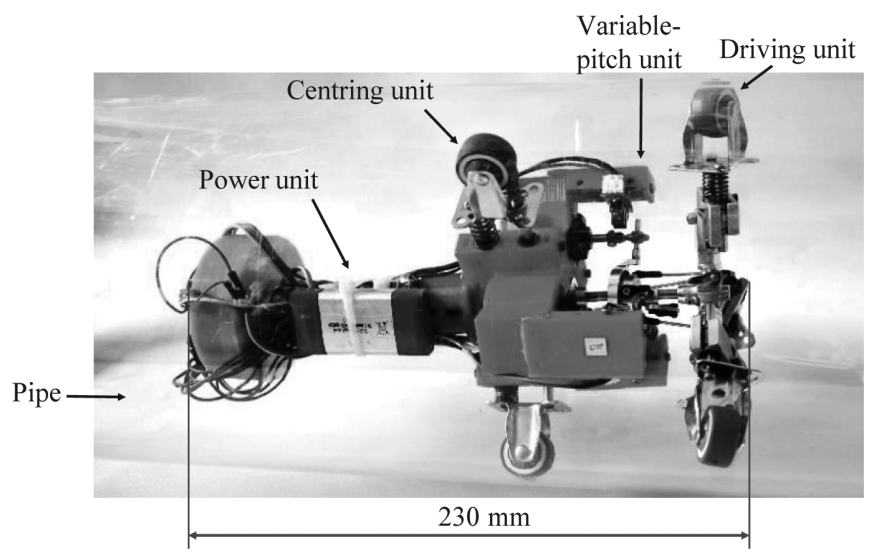

Figure 10. A prototype of variable pitch helical in-pipe robot.

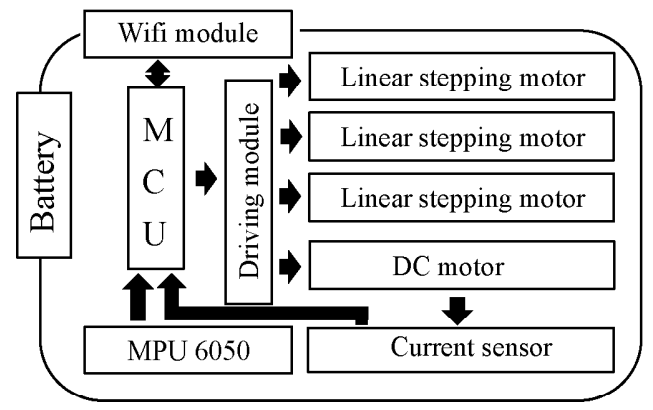

Figure 11. Diagram of the control system.
Figure 12(a)-12(c) illustrate the variable pitch adjusting mechanism and the three states of motions (normal motion, variable speed motion, and variable pitch helical turning motion), as described in Section 3.3.

\subsection{Variable Speed Motion}

Two comparative experiments are performed to verify variable speed motion in the pipe. The helical angle of the robot shown in Fig. 13(a)-13(c) is $5^{\circ}$, whereas that in Fig. $13(\mathrm{~d})-13(\mathrm{f})$ is $45^{\circ}$. The robot is driven to move forward in a Plexiglas pipe within $4 \mathrm{~s}$ with different helical angles. As shown in Fig. 13, the robot with the larger helical angle moves faster than the one with the smaller angle.

\subsection{Turning Motion}

Turning motion experiments are conducted to verify the correctness and validity of the variable pitch working principle and the variable pitch adjusting mechanism. The three helical angles of the robot are initially equal in the straight pipe. When passing through the curved pipe, the three linear stepping motors are controlled to obtain different elongations. Thus, the outer ring on the central axis inclines. The inclination angle of the driving wheel increases on the outer side of the pipe and then decreases when approaching the inner side. The robot successfully passes through the curved pipe with $135^{\circ}$, as shown in Fig. 14.

Figure 15 shows the comparison between constant pitch and variable pitch when the robot is passing through a curved pipe. The angle data are collected by the threeaxis gyroscope of MPU 6050 to show the posture of the prototype. As is shown at $5 \mathrm{~s}$ to $6 \mathrm{~s}$ in Fig. 15(a), severe vibration and slippage happens when the prototype with constant pitch is passing through a curved pipe. It is easy to get stuck in curves. As is shown in Fig. 15(b), the prototype with variable pitch moves quite smooth and stable when passing through a curved pipe. This experiment shows that the novel variable pitch helical in-pipe robot exhibits better turning flexibility and stability in curved pipes.

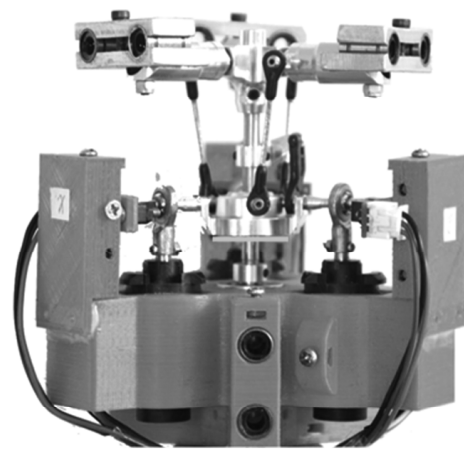

(a)

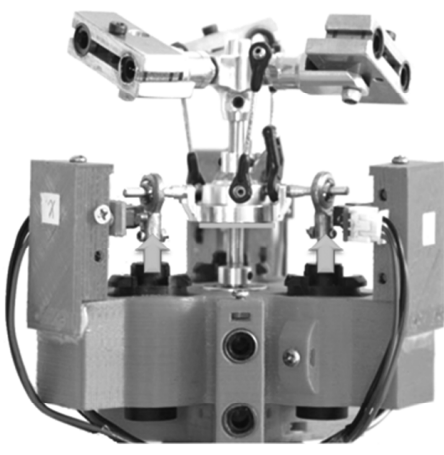

(b)

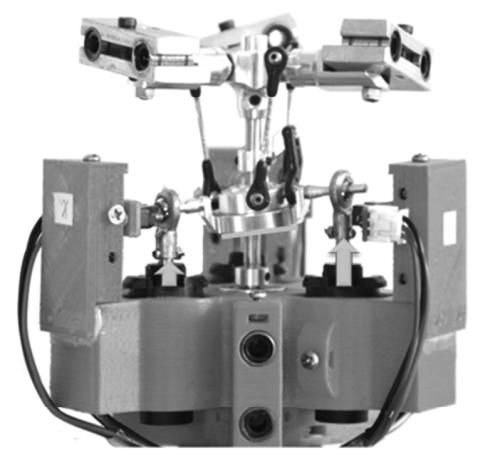

(c)

Figure 12. Three states of motions: (a) normal motion; (b) variable speed motion; and (c) variable pitch helical turning motion. 


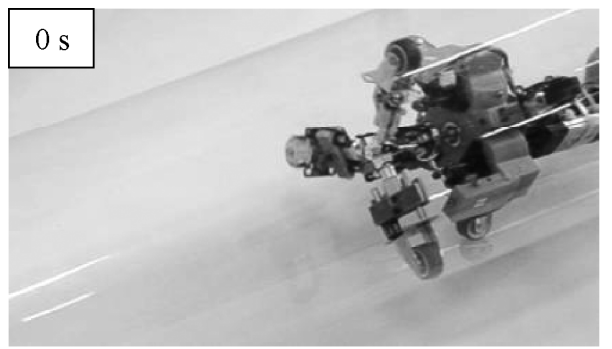

(a)

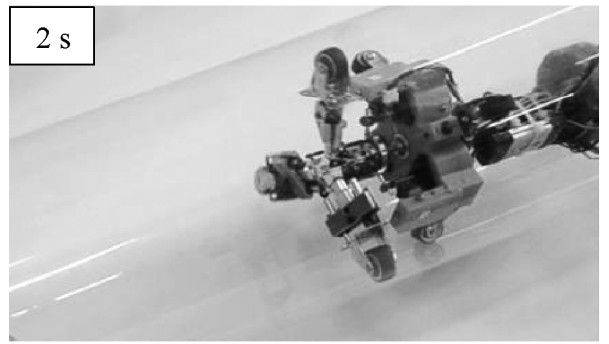

(b)

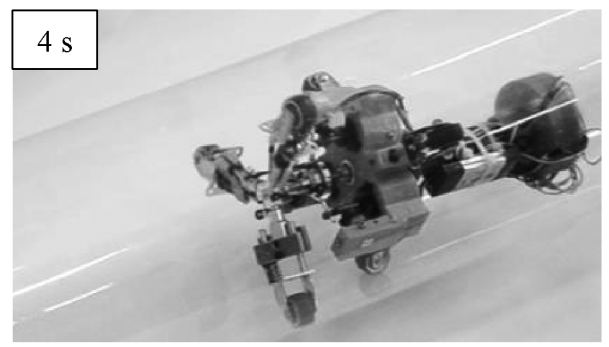

(c)

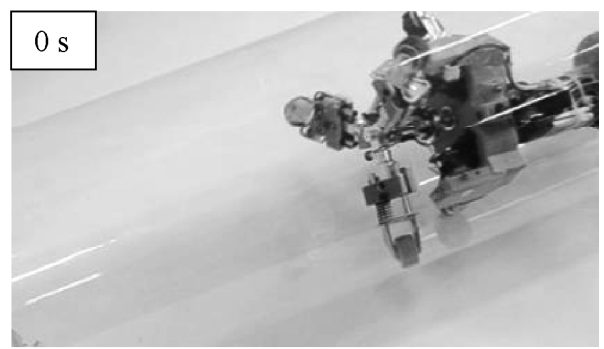

(d)

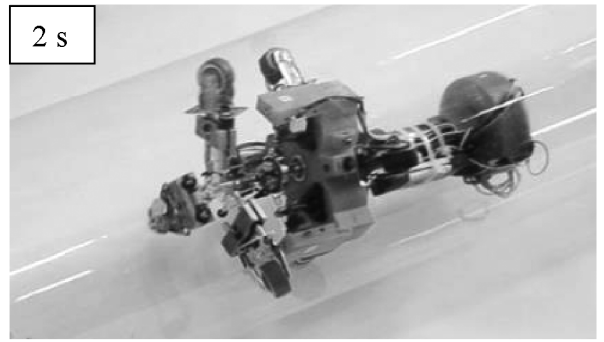

(e)

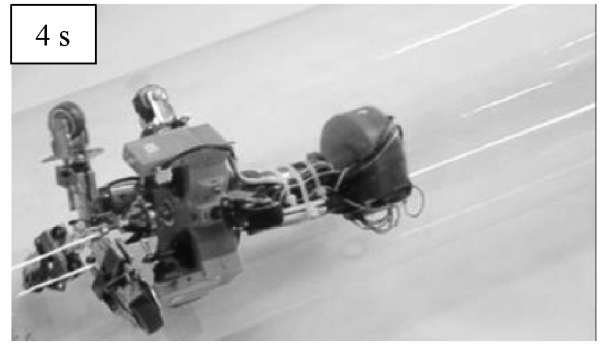

(f)

Figure 13. Variable speed motion of two helical angles: (a) to (c) $5^{\circ}$ and (d) to (f) $45^{\circ}$.

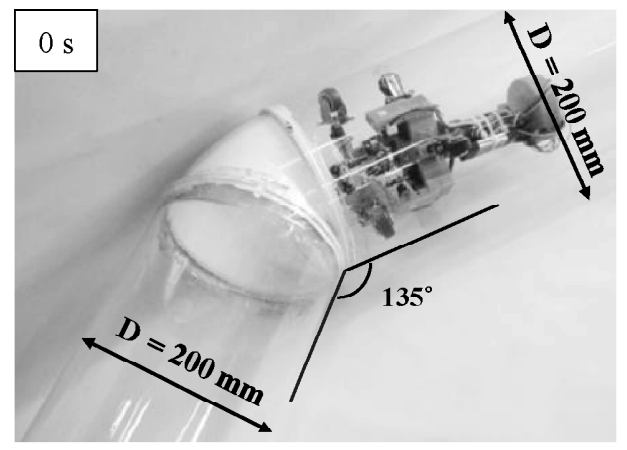

(a)

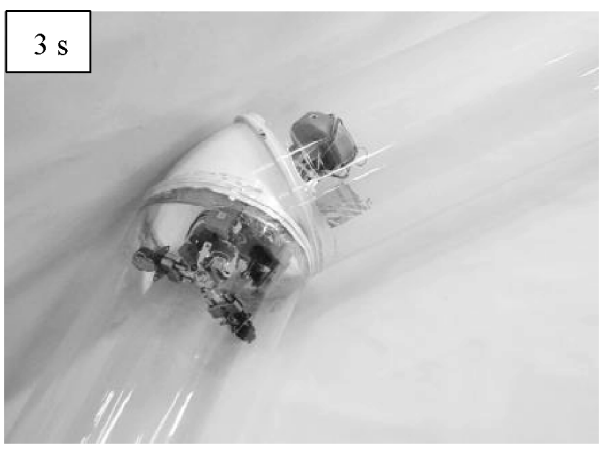

(b)

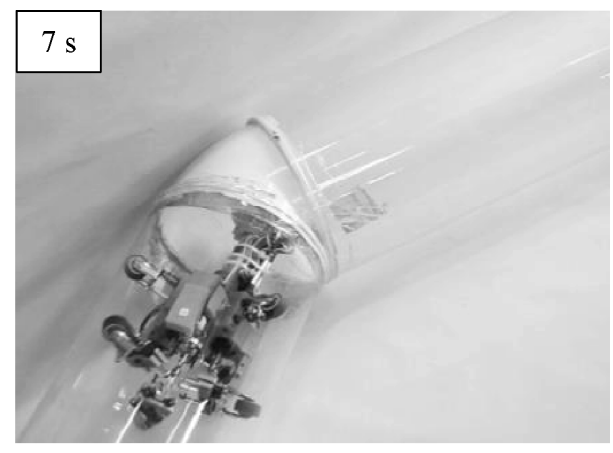

(c)

Figure 14. Turning motion: (a) before entering a curved pipe; (b) in a curved pipe; and (c) pass through a curved pipe successfully.

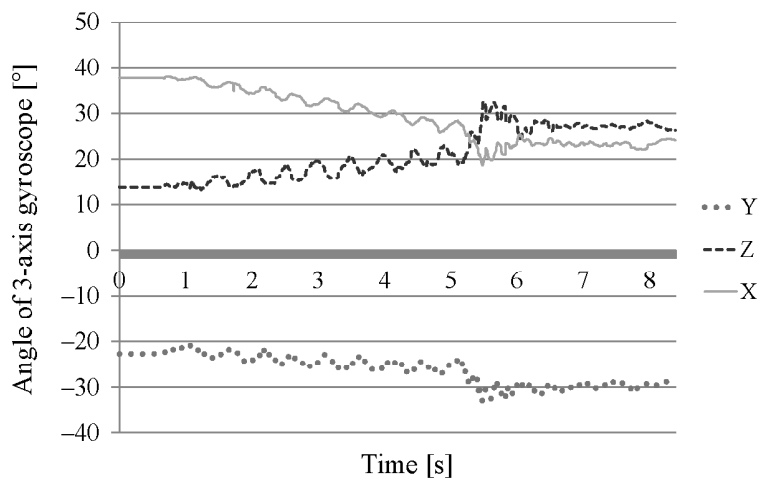

(a)

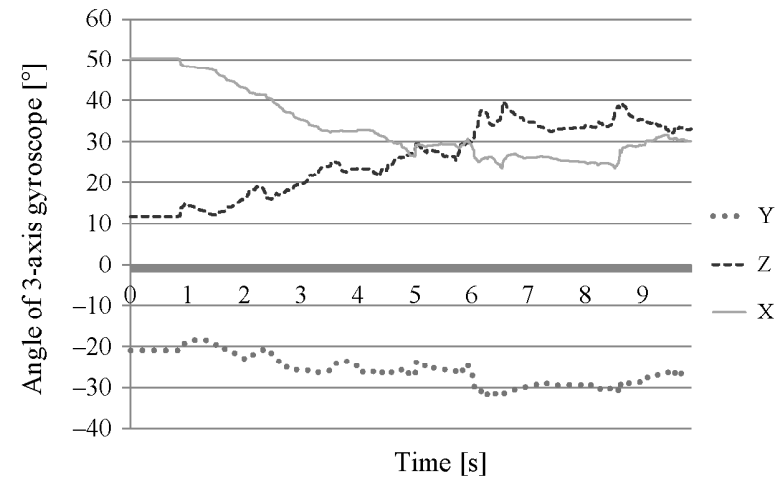

(b)

Figure 15. Angle of 3-axis gyroscope when passing through a curved pipe: (a) constant pitch and (b) variable pitch. 


\section{Conclusion}

In this study, we propose a novel concept of variable pitch helical drive in-pipe robot that can actively pass through curved pipes by calculating and controlling the helical angle of each driving wheel separately.

(1) Unlike the passive tuning mechanism of in-pipe robots reported previously, the different moving speeds of the driving wheels enable the proposed robot to travel through curves actively and flexibly.

(2) This robot can switch between three motion patterns (normal, variable speed, and turning) by controlling variable pitch adjusting mechanism.

(3) The kinematics of the proposed variable pitch helical drive in-pipe robot is analysed by comparing with the kinematics of a typical constant pitch screw drive inpipe robot, and the space spiral trajectories of the two types of robot are drawn.

(4) A prototype is developed to demonstrate the concept and kinematics research. Experiments with variable speed motion and turning motion are conducted successfully.

Future works will emphasize elbow automatic detection through a visual method, an acoustic method, or a posture algorithm. The precise control of helical angles that correspond to different pipe-bending radii will also be the focus of future works. Experiments on different pipe-bending angles must also be conducted. Potential application of the proposed robot could be inspection and maintenance in complex oil and gas pipeline grids, and the nuclear small bore pipes.

\section{Acknowledgement}

The authors would like to thank Sichuan Province of China Science and Technology Support Program (Project Code: 2013GZ0150 and 2014GZ0121) for their support of this paper.

\section{References}

[1] W. Guangrong, M. Peisun, L. Yanming, C. Xi, and S. Hong, The mobile robot for outer surface inspection of boiler tubes, International Journal of Robotics and Automation, 19(1), 2004, 36-41.

[2] G. Granosik, Hypermobile robots - the survey, Journal of Intelligent \& Robotic Systems, 75(1), 2014, 147-169.

[3] J. Tiratsoo, The ultimate guide to unpiggable pipelines, (Melbourne, Victoria, Australia: Pipelines International, 2013).

[4] A. Shukla and H. Karki, Application of robotics in onshore oil and gas industry - a review Part I, Robotics and Autonomous Systems, 75, 2016, 490-507.

[5] I.N. Ismail, A. Anuar, K.S.M. Sahari, M.Z. Baharuddin, M. Fairuz, and J.M. Saad, Development of in-pipe inspection robot: A review, Sustainable Utilization and Development in Engineering and Technology (STUDENT), IEEE Conference on, 2012, 310-315.

[6] N.S. Roslin, A. Anuar, M.F.A. Jalal, and K.S.M. Sahari, A review: hybrid locomotion of in-pipe inspection robot, Procedia Engineering, International Symposium on Robotics and Intelligent Sensors, 41, 2012, 1456-1462.

[7] H.P. Huang, J.L. Yan, and T.H. Cheng, Development and fuzzy control of a pipe inspection robot, Industrial Electronics, IEEE Transactions on, 57(3), 2010, 1088-1095.

[8] A.A. Nassiraei, Y. Kawamura, A. Ahrary, Y. Mikuriya, and K. Ishii, Concept and design of a fully autonomous sewer pipe inspection mobile robot "KANTARO," Robotics and
Automation, 2007 IEEE International Conference on, IEEE, 2007, 136-143.

[9] E.C. Dertien, Design of an inspection robot for small diameter gas distribution mains, Ph.D. thesis, University of Twente, 2014.

[10] T, Nishihara, K. Osuka, and I. Tamura, Development of a simulation model for inner-gas-pipe inspection robot: SPRING, Proceedings of SICE Annual Conference, 2010, 902-904.

[11] D. Tang, Q. Li, S. Jiang, Z. Deng, and H. Liu, Design and analysis of a pipeline robot with the function of differential movement, Jixie Gongcheng Xuebao (Chinese Journal of Mechanical Engineering), 47(13), 2011, 1-8.

[12] H.M. Kim, Y.S. Choi, H.M. Mun, S.U. Yang, C.M. Park, and H.R. Choi, 2-2D differential gear mechanism for robot moving inside pipelines, In Intelligent Robots and Systems (IROS), 2015 IEEE/RSJ International Conference on, 2015, 1152-1157.

[13] J. Borenstein, M. Hansen, and A. Borrell, The OmniTread OT-4 serpentine robot - design and performance, Journal of Field Robotics, 24(7), 2007, 601-621.

[14] Q. Liu, T. Ren, and Y. Chen, Characteristic analysis of a novel in-pipe driving robot, Mechatronics, 23(4), 2013, 419-428.

[15] Q. Liu, Y. Chen, T. Ren, and Y. Wei, Optimized inchworm motion planning for a novel in-pipe robot, Proceedings of the Institution of Mechanical Engineers, Part C: Journal of Mechanical Engineering Science, 228(7), 2014, 1248-1258.

[16] R. Tao, Y. Chen, and L. Qingyou, A helical drive in-pipe robot based on compound planetary gearing, Advanced Robotics, 28(17), 2014, 1165-1175.

[17] J.M. Mirats Tur and W. Garthwaite, Robotic devices for water main in-pipe inspection: A survey, Journal of Field Robotics, 27(4), 2010, 491-508.

[18] M.M. Moghaddam, M. Arbabtafti, and A. Hadi, In-pipe inspection crawler adaptable to the pipe interior diameter, International Journal of Robotics and Automation, 26(2), 2011, 135.

[19] M. Horodincă, A. Preumont, and I. Burda, The heli-pipe inspection robots architecture for curved pipes, 2003, 155-160.

[20] T. Li, S. Ma, B. Li, M. Wang, and Y. Wang, Axiomatic design method to design a screw drive in-pipe robot passing through varied curved pipes, Science China Technological Sciences, 59(2), 2016, 191-202.

[21] A. Kakogawa and S. Ma, Experimental verification of analytical torques enabling a screw drive in-pipe robot to pass through bent pipes, Robotics and Biomimetics (ROBIO), 2011 IEEE International Conference on, IEEE, 2011, 1742-1747.

[22] T. Nishimura, A. Kakogawa, and S. Ma, Pathway selection mechanism of a screw drive in-pipe robot in T-branches, Automation Science and Engineering (CASE), 2012 IEEE International Conference on, IEEE, 2012, 612-617.

[23] A. Kakogawa and S. Ma, Stiffness design of springs for a screw drive in-pipe robot to pass through curved pipes and vertical straight pipes, Advanced Robotics, 26(3-4), 2012, 253-276.

[24] M. Horodinca, I. Doroftei, E. Mignon, and A. Preumont, A simple architecture for in-pipe inspection robots, Proc. Int. Colloq. Mobile, Autonomous Systems, Magdeburg, Germany, June 2002, 61-64.

[25] T. Kamegawa, T. Baba, and A. Gofuku, V-shift control for snake robot moving the inside of a pipe with helical rolling motion, Safety, Security, and Rescue Robotics (SSRR), 2011 IEEE International Symposium on IEEE, 2011, 1-6.

\section{Biographies}

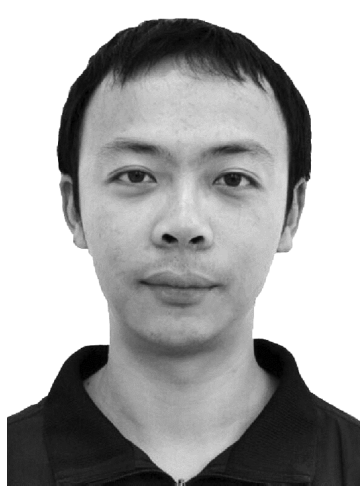

Tao Ren received his B.E. and M.E. from the School of Mechatronic Engineering, Southwest Petroleum University, Sichuan Province, China, in 2011 and 2014, respectively. He is currently working towards his Ph.D. in the School of Mechatronic Engineering. His research interests include the oil and gas in-pipe inspection robot and the downhole tractor. 


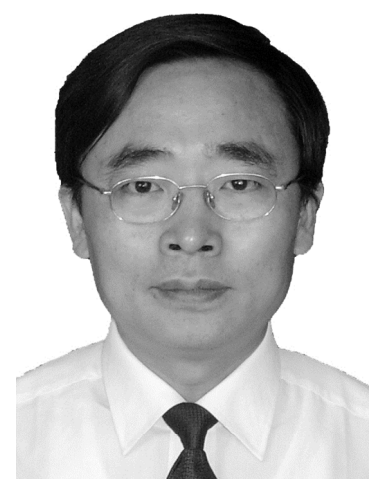

Qingyou Liu received his Ph.D. from the School of Mechatronic Engineering, Southwest Petroleum University, Sichuan Province, China, in 1997. From 1997 to 1999, he was a Post-Doctoral candidate in The State Key Laboratory of Mechanical Transmission, Chongqing University, Chongqing, China. Currently, he is a professor in the Key Laboratory of Fluid and Power Machinery, Ministry of Education, Xihua University, Sichuan Province, China. His research interests include the oil and gas equipment, oil and gas wells engineering mechanics, and in-pipe inspection robot.

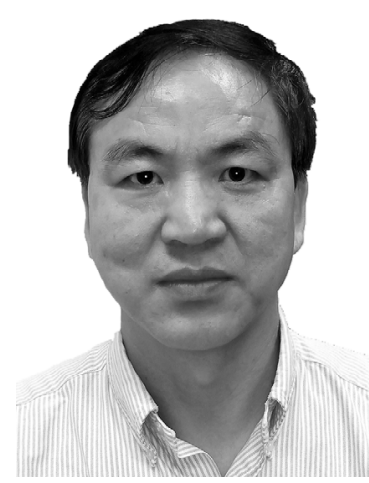

Yonghua Chen received his Ph.D. from the School of Engineering, University of Liverpool, Liverpool, England, in 1990. Currently, he is a professor in the Department of Mechanical Engineering at The University of Hong Kong, Hong Kong, China. His current research interests include engineering design, rapid prototyping and robotic machining, haptic modelling and computeraided medical surgeries. He is a chartered engineer and a member of the Institution of Mechanical Engineer. He is also an active IEEE member who has chaired 4 IEEE international conferences. He has published over 100 referred papers in international journals and conferences in the areas of machine vision, computer-aided design and manufacturing.

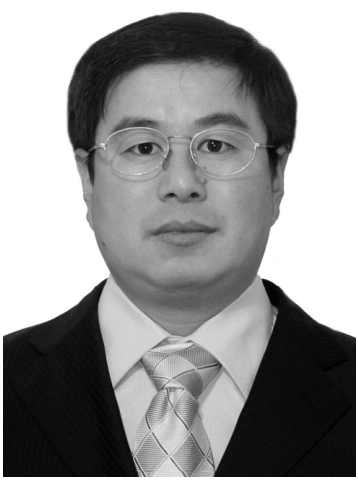

Shouhong Ji received his B.E. from the School of Mechatronic Engineering, Southwest Petroleum University, Sichuan Province, China, in 1992. He is currently working towards his MEng in the College of Chemistry and Chemical Engineering. Currently, he is an engineer in Zhejiang Zhenergy Natural Gas Operation CO., LTD, Zhejiang, China. His research interests include oil and gas storage and transportation safety production management, maintenance and repair technology, pipeline integrity technology, and pipeline corrosion mechanism. 